\title{
Stereo-PIV measurements in turbulent rotating convection
}

\author{
R.P.J. Kunnen ${ }^{1}$, B.J. Geurts ${ }^{1,2}$, and H.J.H. Clercx ${ }^{1,2}$ \\ 1 Dept. of Physics \& J.M. Burgerscentrum, Eindhoven University of Technology, \\ P.O. Box 513, 5600 MB Eindhoven, The Netherlands, R.P.J.Kunnen@tue.nl \\ 2 Dept. of Applied Mathematics \& J.M. Burgerscentrum, University of Twente, \\ P.O. Box 217, 7500 AE Enschede, The Netherlands
}

Convection and rotation are prevalent influences on many geophysical flows, such as the flows in the atmosphere and in the oceans. Also in turbo-machinery and chemical process engineering such situations arise. A convenient model for studying these effects is provided by rotating Rayleigh-Bénard convection (RRBC) in a cylindrical container: a fluid enclosed by a rotating cylinder is heated from below and cooled from above.

RRBC is geverned by several dimensionless parameters: the Rayleigh number $R a=g \alpha \Delta T H^{3} /(\nu \kappa)$, the Taylor number $T a=\left(2 \Omega H^{2} / \nu\right)^{2}$, the Prandtl number $\sigma=\nu / \kappa$, and, the geometry aspect-ratio $\Gamma=D / H$. Here $D$ and $H$ are diameter and height of the cylinder, $g$ is the gravitational acceleration, $\Delta T$ the temperature difference between the plates, $\Omega$ the rotation rate antiparallel to gravity, and $\alpha, \nu, \kappa$ are thermal expansion coefficient, kinematic viscosity and thermal diffusivity of the fluid, respectively.

Here we present results from an experimental study of RRBC. Stereoscopic particle image velocimetry (SPIV) is applied [1]. This measurement technique provides the three velocity components as measured in a planar cross-section of the flow domain. The setup is displayed schematically in Fig. 1. A closed plexiglas cylinder of height and diameter $H=D=23 \mathrm{~cm}(\Gamma=1)$ is filled with water seeded with small polyamid tracer particles. A laser light sheet illuminates a horizontal cross-section of the cylinder at half-height. Two cameras are mounted above the cylinder at off-axis angles, to record the particle motions in the light sheet plane. All parts, including cooling and heating units for the cylinder, are placed on a rotating table. The experimental set-up allows to monitor the flow in a window of roughly $9 \times 12 \mathrm{~cm}$.

In the measurements constant $R a=1.1 \times 10^{9}$ and $\sigma=6.4$ have been used. The measurements were conducted in two different rotation regimes: the first at very small rotation-rates $\left(0 \leq \Omega \lesssim 0.03 \mathrm{rad} / \mathrm{s}, 0 \leq T a \lesssim 2 \times 10^{7}\right)$, and the second at larger rotation rates (up to $T a=2.2 \times 10^{10}$ ).

At low rotation-rates the entire domain is filled by a so-called large-scale circulation (LSC). Hot plumes rise on one side of the cylinder, while cold 

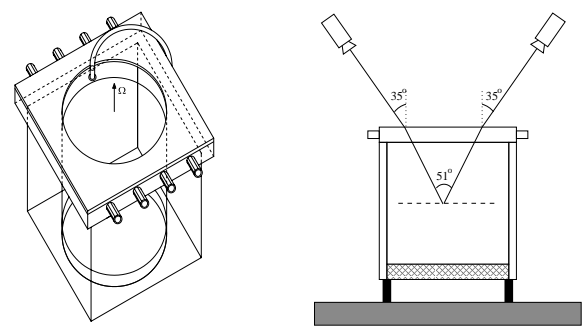

Fig. 1. Sketches of the experimental set-up and the positioning of the cameras. A square box filled with water surrounds the cylinder to ease optical access. The water circulation chamber on top (with temperature sensor) is used to cool the cylinder from above, while still being transparent.

plumes sink on the other. The LSC displays dynamics on widely varying time scales, from an azimuthal oscillation with period of order one minute to sudden rotations and cessations at time intervals of several hours to a day [2]. The azimuthal oscillation is investigated as follows. In the interrogation plane the LSC is organized into a few large parts of the flow domain with either positive or negative vertical velocity $w$, cf. the velocity snapshot in Fig. 2(a). The centroid of $w$ in either the $w>0$ or $w<0$ regions is computed. The angle $\phi$ between the positive $x$-axis and the line connecting these centroids is taken as the orientation of the LSC. The centroids and the connecting line are also indicated in Fig. 2(a). The time history and autocorrelation of the orientation $\phi$ is shown in Fig. 2(b). The peaks in the autocorrelation directly provide the oscillation period $\tau_{0}=(1.4 \pm 0.1) \times 10^{2} \mathrm{~s}$, which, nondimensionalized as $H^{2} /\left(\kappa \tau_{0}\right)=(2.6 \pm 0.2) \times 10^{3}$, matches well with the result $H^{2} /\left(\kappa \tau_{0}\right)=0.084 R a^{0.50 \pm 0.01}=2.8 \times 10^{3}$ of [2]. At very small but nonzero rotation rates the LSC displays an anticyclonic precession, this motion is currently under study.

(a)

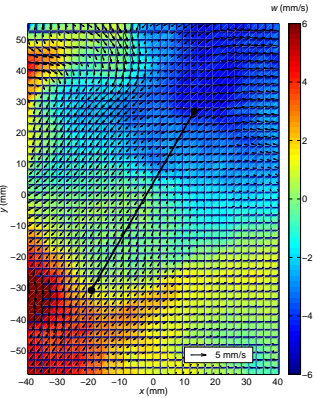

(b)

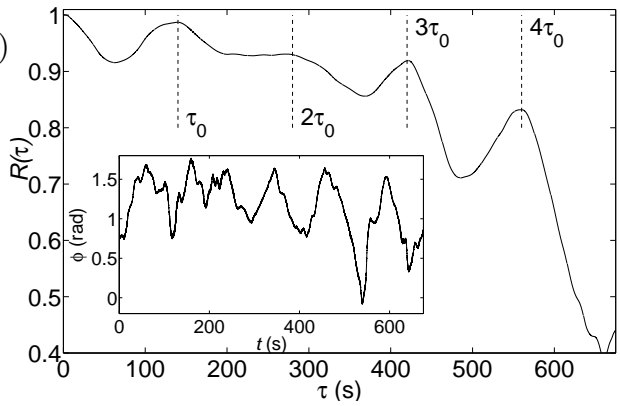

Fig. 2. (a) Example velocity snapshot. Arrows represent the horizontal component, while the greyscale is for the vertical component. The centroids and the orientation line are also included. (b) Autocorrelation $R(\tau)$ of the LSC orientation $\phi$. Inset: time history of $\phi$. 

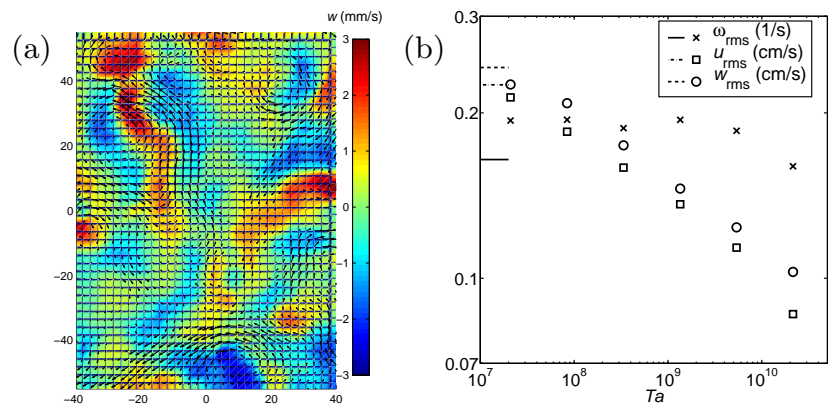

Fig. 3. (a) Velocity snapshot at $T a=2.2 \times 10^{10}$. (b) Root-mean square velocity $\left(u_{\mathrm{rms}}, w_{\mathrm{rms}}\right)$ and vorticity $\left(\omega_{\mathrm{rms}}\right)$ as a function of $T a$. The line segments on the vertical axis indicate the rms values at $T a=0$.

The regime of strong rotation covers the Taylor numbers in the range $10^{7} \lesssim$ $T a \lesssim 2 \times 10^{10}$. From velocity snapshots it can be concluded that horizontal length scales decrease as rotation is increased. The convective plumes now contain considerable vorticity. Fig. 3(a) displays a velocity snapshot at the highest $T a=2.2 \times 10^{10}$ used. The attenuating effect of rotation on the turbulence intensities can be observed in Fig. 3(b). The horizontal and vertical rms velocity fluctuations are plotted as a function of $T a$, along with the rms value of the vertical vorticity component. These rms values are averaged over the measurement area and in time. Both horizontal and vertical rms velocities become smaller under rotation, with a power-law drop-off that scales as $T a^{-0.13 \pm 0.01}$. The rms vorticity is nearly constant at a higher value than at $T a=0$. Only at very large $T a$ this value shows a decrease.

In this experiment the LSC of nonrotating convection was characterized independently, based on SPIV measurements. The oscillation frequency matched well with previous results. The addition of a very small background rotation causes anticyclonic rotation of the LSC; this is currently under study. At higher rotation rates the LSC has fragmented into small, but intense vortical regions. We observed a decrease of the turbulence intensities as well as a decreased horizontal length scale. Further investigations will include statistics of the vortical plumes and properties of the convective turbulence, such as structure functions.

The authors wish to thank the Foundation for Fundamental Research of Matter (Stichting voor Fundamenteel Onderzoek der Materie, FOM) for financial support.

\section{References}

1. M. Raffel, C. Willert, J. Kompenhans: Particle Image Velocimetry, (Springer, Berlin 1998) pp 174-184

2. H.-D. Xi, Q. Zhou, K.-Q. Xia: Phys. Rev. E 73, 056312 (2006) 